International Journal of Engineering \& Technology, $7(2.28)(2018) 157-159$
International Journal of Engineering \& Technology
SPC
Website: www.sciencepubco.com/index.php/IJET
Research paper

\title{
A Decision Phases of a Supply Chain Management : A Proposed Decision Support System to Boost Organizational Decision Making
}

\author{
Desmond D.K. Attadjei ${ }^{1}$, Yash Madhwal ${ }^{1}$, And Dr. Peter B. Panfilov ${ }^{1 *}$ \\ ${ }^{I}$ School of business Informatics, Faculty of Business and Management, National Research University-Higher \\ School of Economics, 20 Myasnitskaya St., 101000 Moscow, Russia. \\ *Email:desdejei@gmail.com:
}

\begin{abstract}
A powerful and effective store network administration sires, a smooth running of a business, quality items or potential benefits, consequently escalating client trust and business picks up. To land at this phase in an association's business outgrowth, all rely upon the best choices that must be taken to move the correct way. These choices are normally aftereffects of past events and outside information that have been incorporated to give data or learning. For this reason, a decision support system is hereby proposed to be able to pool data from the 3 main decision phases of a Supply Chain Management, namely: (1) supply chain design (Strategy) phase, (2) decision in supply chain management (Planning) phase, and (3) operational level (Operation) phase. This proposed framework is pointed and boosting basic leadership process, by giving brought together data to help the hierarchical basic leadership process.
\end{abstract}

Keywords: Decision Support Systems; Decision Phases; Decision Model; Supply Chain Management.

\section{Introduction}

Supply Chain Management (SCM) fills in as an indispensable section of any organization, it is a reliant or autonomous system of a stream of material or data coming from supplier to end customers [1]. With globalization, the trade business has depended on the supply chain management by bringing the world nearer through the cooperation and coordination among different bodies of the logistics. A supply chain management requires numerous choices identifying with the stream of data, item and assets. Every choice ought to be made to raise the store network excess. [2]. Decision Support Systems (DSS) are systems put in place to help stakeholders of a supply chain to take informed decisions based on information or data generated from part or the entire supply chain pipeline. Its significance in every line of business or the commodity flow pipeline could not be underrated [3]. There are various risks and uncertainties that are associated with a supply chain at one point or the other [4] .Some of these associated risk may be openly and readily visible while others may need technical or critical processing of information that may be hidden in complex data [5]; hence the significance of a DSS to help mitigate some of these risks. The adoption of the DSS in the supply chain also avails to stakeholders the chance to be able to automate processes, eliminating the high rate of errors associated with entire human interventions; it can further help reduce cost by way of helping to consolidate the demand and supply indicators that control the flow of goods or services in the supply chain pipeline.

\section{Decision Process in Supply Chain Manage- ment}

During supply chain management the change from pre-arranging (the phase that happens before an inventory network is conveyed) to start (the primary stages in the genuine sending of the store network), confront choices difficulties, for example, distinguishing the particular conditions that trigger the requirement for progress and planning continuous inventory network stream while at the same time reconfiguring the engineering of that production network. Each decision phases have different roles and time working duration. Every decision phase has a distinctive role and time span, which ought to be made more viable when worked with existing accessible information for each stage. Fig1. Shows the basis supply chain decision phases.

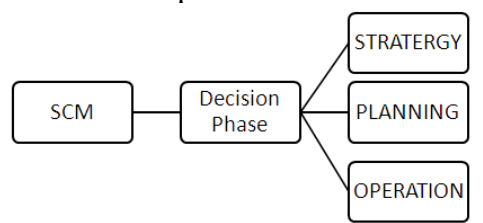

Fig. 1: Supply Chain Management Decision Phases

\subsection{Supply Chain Strategy}

This phase decides the overall structure of the supply chain for the following couple of years, this phase is set at the management level for long term and can be high-cost to firm if alternation stands in need in short notice. This phase decides the overall con- 
figuration of the chain a, deciding its components, resource allocation, outsources or in-house. Firms aims at increasing supply chain surplus [6] by configuration support of its strategic objectives. To avoid short term alternation, company must take into account all the uncertain possibilities in market over the following few years.

\subsection{Supply Chain Planning}

The time frame is considered according to demand and supply which can be from a quarter to a year. Constraints that are analyzed during the strategy phase can help to generate supply chain surplus. This phase includes it all, starting from predicting the market demand to which market will be provided the finished goods to which plant is planned in this stage. All the participants or employees involved with the company should make efforts to make the entire process as flexible as they can [7].

\subsection{Supply Chain Operation}

This phase works on the weekly or day to day activity with short timeframe, weekly, daily or hourly working with minimum demand uncertainty. They work on individual customer orders, i.e. setting delivery schedule, generate pickup list dealing with fixed policies and planning. The organizations that do error is sudden bouncing to this level without experiencing past levels.

\section{Decision Support System}

Decision support systems are systems which are basically made up of some specific parts that need drives this system consist of the language system (LS)- which deals with the instructions or contents that the system is capable of accepting, an aspect that has the capability or the responsibility to display information the DSS wants show (presentation system (PS)), the knowledge system (KS), consisting of the information or data and or knowledge that the system contains for analysis; and the problem-processing system (PPS) made of the set of tools or software engine that drives the system by trying to realize and find solutions to the problem at hand. All of these components of the system tend to work hand-inhand and therefore have to understand each other in order to function appropriately to provide the required or expected support they are meant for

However, these systems do not act or function in isolation, as they demand human or device interaction to be able to input data, instruction or issue queries to the system [8] [9] [10]. Below (Fig 2.) is a simple illustration of a generic DSS and how its components interact and relate to each other.

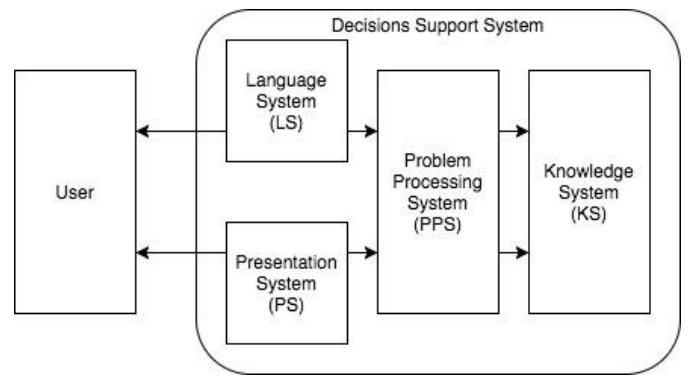

Fig. 2: Generic Decision Support System

\subsection{General Description of System Functioning}

The users who interact with the system represent a varied group of individuals or devices that feed data into the system or instruct the system to perform some problem solving tasks or modify system knowledge. Such feeds or requests are made using the LS component of the DSS. The LS component sends instruction to the PPS component, which is the driving engine of the system to be processed. For the PPS to process element from the LS, it may be required to make some knowledge or data acquisition from the KS stores and other relevant sources like a user or other devices. After the KS has provided the PPS with all the required information and or knowledge for it to perform all the instructions given it, it sends or displays responses to the user by the help of the PS [11].

\section{Decision Support System For Supply Chain Management}

\subsection{Proposed Model}

The proposed model (fig. 3) depicts an iterative block of a decision support system that could be applied in the supply chain management process. The entire process begins with the process of data acquisition from the various stages of the supply chain. This stage deals with securing clean data sets with the optimum quality for the variables that need to be measured by the system. This can be made up quantitative and qualitative data depending on what decision the system needs to take and its data requirement. Once data is sourced into the system, they are cleaned and processed in a required way and stored into a database that will await further usage or processing.

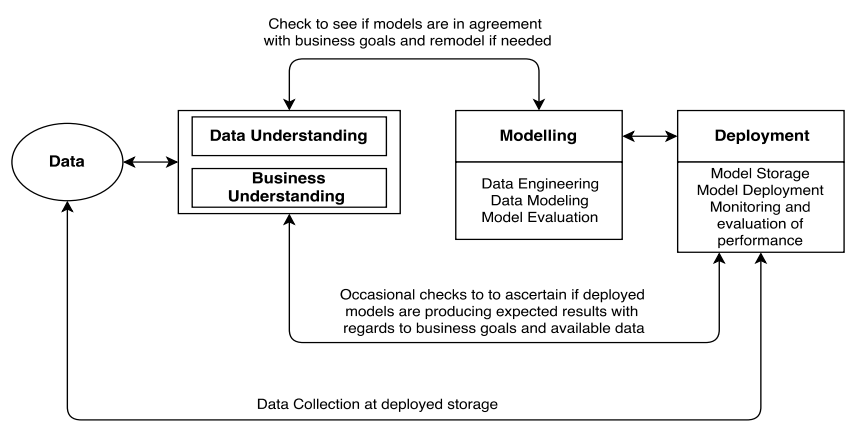

Fig.3. Proposed Model of Decision Support System

The next step in the processing is for the user or decision makers to understand the data that has been stored in the system. The user must be able to understand whether the data in store would be able to achieve its purpose and whether variables of the data are in line with achieving set goals or objectives. If there is the need, more data is sourced or requested in an iterative manner until expected solutions are reached. Understanding the data and the business must work hand-in-hand in order to collect the right type of data.

Once a consensus is reached at the point of understanding the business and the data, the next stage is to model the data or process the data to help solve the problem at hand or generate a backing for decisions being reached. The modelling stage deals with the feature engineering of the data to be able to fit into the model that would be used. Once data is fully engineered, model building becomes the next procedure, which involve applying various models to the data with the required parameters and evaluating them as well. This gives a chance to be able to settle on a robust model by the system for deployment. Model building stage will also need a coordination between understanding the business and the data to be sure the model being used are appropriate for the business and the available data. This will also require an iterative process if need be.

The final block of the process is the deployment stage where the built models or solution are deployment to the decision makers or the stakeholders to be used to support the supply chain decision making process. This stage has to do with storing obtained model which could be reused or consulted at a later time for improvement or auditing. As the model or solution is deployed they are being monitored and evaluated to score its performance right from its usage. Data is however collected at this stage as well into the data storage system to be able to make adjustment or changes 
were needed. Deployed models are also monitored to be sure they are providing the solution that are in line with the supply chain goals or objectives; when there is a need, models will either be done again or may be discarded for the whole process to be carried out again. This process describes how each block of the system cannot stand in isolation as it is also depicted in Holsapple's system [11]; making it necessary for all parts of the block to have a mutual language of communication to enable the system function to the optimum stage and to provide the right solution.

\subsection{Technical Description of System Operation}

At each block, the objectives and goals are defined with explicit and enough details. Processes also accumulate all necessary data that is generated and would be needed in the event of making decisions. This could be in the form of text details as well as numerical representations. Once there is enough data, and detailed goal and objective descriptions, the accumulated data is ready to be linked to the objective of the particular chain involved.

With the proposed system comes a semantic technology embedded in each block of the supply chain. This technology is required to link the semantics of the objective/goals of the block to the accumulated data. The semantic technology that is applied would generate supportive decisions or suggestions from the links created between the two set of information given it. These suggestions expected to be made by the system are reached by way of using specified rules based on stakeholder preferences of the chain optimization. Suggestions would be are more detailed report rather than just a simple conditional output. This would be able to make the system provide a wide range of suggestive outputs instead of limited or restricted ones. Therefore, a combination of different factors or variables from the analyzed data helps to produce this decision support information.

Once the data being accumulated in the business (supply chain block) process changes with time, the semantic model underlying the system is also adjusted (updated) to meet the needs and requirements as such. This is done with a specified interval proposed by stakeholders; to promote a robust and up-to-date system giving rise to effectiveness and efficiency. Updates are usually procedures that are expected to planned and fixed by stakeholders of the entire chain based on individual organizational or departmental milestones or preferences, considering needed resources This could depend on the type of supply chain (business) being operated, the rate and seasons of change in the associated with the supply chain, and other related variables that has the tendency to influence the model building process or the overall objective.

Along with the semantic model also operates a predictive or forecasting model that would be applied to the data. This would also help make forecasting or predictions based on the data and the goal of the particular block where it is being adopted. For instance, the model could forecast of supplies that would be demand in a particular season or by particular customers/clients, given the historical data that have been accumulated over some period of time. These two models (semantic \& predictive/forecast models) which work in parallel do so with the aim of complimenting results produced from each other as they both function on the same, similar or related data sets; and are expected to produce results towards the same goals and objectives of that supply chain block. Working in parallel and towards the same objectives, we believe these models possess a tendency to provide robust reliable result.

The functioning of these two models are done in parallel on each individual block in the chain (plan strategy...) and results/outputs are then transmitted to the final decision phase of the system which are also unified by way of also applying another semantic model to produce a single report, also taking into consideration the larger goal and objective of the final decision goals and objectives. These goals and objectives which are usually bound to change from time to time are usually dependent on decisions the stake- holders of the supply wish or hope to reach or achieve at a particular point in time, upon system query. A generated unified result or out will now be subjected to stakeholder review and scrutiny to be sure they are within the context of the situation or issues at hand, or whether propositions made are feasible or practical enough, before implementation.

\section{Conclusion}

Decision Support System unifies this system with every stage of the supply chain and henceforth, management becomes much easier and less engulfed with unnecessary human errors and thereby cutting cost, to be invested in other necessary sectors of the business. The main three decision phases of supply chain model i.e. Strategy, Planning and Operation, can rely on this DSS approach, and can be rectified with the proposed system of DSS model.

\section{Acknowledgement}

This research was partially supported by the National Research University Higher School of Economics (HSE) of Moscow, Russia.

\section{References}

[1] Supply Chain Resource Cooperative, "Poole College of Management," [Online]. Available: https://scm.ncsu.edu/scm-articles/article/what-issupply-chain-management.

[2] S. Chopra, P. Meindl and D. V. Kalra, "Supply Chain Management," in Stratergy, Planning, and Operation, vol. 6, Pearson, 2016.

[3] V. Sauter, Decision Support Systems for Business Intelligence, vol. 4, John Wiley \& Sons, 2014, 2014, p. 453.

[4] T. C. I. H. Stefan Nickel, "A critical review on supply chain riskDefinition, measure and modeling," Omege, vol. 52, pp. 119-132, April 2015.

[5] F. F. U. K. F. d. J. E. Frederik Hogenboom, "A Survey of event extraction methods from text for decision support systems," Decision Support Systems, vol. 85, pp. 12-22, May 2016.

[6] Wikipedia, "Wikipedia," [Online]. Available: en.wikipedia.org/wiki/Supply_chain_surplus.

[7] tutorialspoint, "tutorialspoint simply learning," [Online]. Available www.tutorialspoint.com/supply_chain_management/supply_chain_ma nagement decision phases.htm.

[8] D. J. S. R. \&. B. F. Power, Decision support systems., John Wiley \& Sons, Ltd. , 2015

[9] S. T. H. S. S. A. K. A. W. M. Afshan Naseem, "Decision support system for optimum decision making process in threat evaluation and weapon assignment: Current status, challenges and future directions," Annual Reviews in Control, vol. 43, pp. 169-187, 2017.

[10] A. K. B. L. S. A. A. K. \&. H. K. Masum, "Intelligent human resource information system (i-HRIS): a holistic decision support framework for HR excellence," International Arab Journal of Information Technology, vol. 15, pp. 121-130, 2018.

[11] C. W. Holsapple, "DSS architecture and types," Handbook on Decision Support Systems, vol. 1, pp. 163-189, (2008). 